\title{
Structural and Secondary Relaxations in Supercooled Di-n-butyl Phthalate and Diisobutyl Phthalate at Elevated Pressure
}

\author{
M. Sekula, ${ }^{\dagger}$ S. Pawlus,${ }^{\dagger}$ S. Hensel-Bielowka, ${ }^{\dagger}$ J. Ziolo, ${ }^{\dagger}$ M. Paluch,${ }^{\dagger,}$ and C. M. Roland ${ }^{*,+}$ \\ Institute of Physics, Silesian University, ul. Uniwersytecka 4, 40-007 Katowice, Poland, and \\ Naval Research Laboratory, Chemistry Division, Code 6120, Washington, D.C. 20375-5342
}

Received: November 26, 2003; In Final Form: February 13, 2004

\begin{abstract}
Broadband dielectric measurements at very large hydrostatic pressures (up to $1.8 \mathrm{GPa}$ ) are used to investigate the effect of molecular structure on the dynamical properties of supercooled di- $n$-butyl phthalate (DBP) and its isomer, diisobutyl phthalate (DiBP). At atmospheric pressure, both the shape of the $\alpha$-relaxation loss peak and the fragility are essentially the same for the two materials, although the behavior of their $\beta$-relaxations differs. The activation energy for the $\beta$-process in DiBP, which is independent of pressure, is larger than that for DBP, while the activation volume of the former is about an order of magnitude larger. In contrast to the similarities of the $\alpha$-relaxation at low pressure, distinct differences between the two isomers become evident at elevated pressure. The activation volume for the $\alpha$-relaxation and the pressure coefficient of the glass transition temperature are significantly larger for DBP than DiBP. On the other hand, the fragility of both liquids is invariant to pressure. This latter observation corroborates recent results from high-pressure viscosity measurements on DBP, and is consistent with the invariance of the $\alpha$-peak breadth to pressure. Related to the large value of the Kohlrausch stretch exponent and its invariance to pressure, there is no decoupling of the $\alpha$-relaxation times and the dc-conductivity over the investigated range of temperatures and pressures. This is consistent with the idea that the decoupling of dynamic variables upon approach to $T_{\mathrm{g}}$ is a consequence of increases in intermolecular cooperativity and dynamic heterogeneity. We also compare herein the characteristic temperatures, and their associated relaxation times, at which various changes in the dynamics become manifest in the DBP and DiBP.
\end{abstract}

\section{Introduction}

Establishing fundamental connections between chemical structure and the physical properties of materials is an ongoing challenge of central importance in both condensed matter physics and material science. An especially useful probe of the molecular motions underlying macroscopic behavior is dielectric spectroscopy, especially when broad-band measurements are carried out as a function of both temperature and pressure. In this manner, the entire supercooled regime of glass-forming liquids and polymers can be investigated. Various groups are actively engaged in the use of pressure as an experimental variable, addressing issues such as the change in dynamics observed above the glass transition temperature, ${ }^{1-6}$ the contribution of volume and thermal energy to relaxation, ${ }^{7-11}$ the role of configurational entropy changes on the dynamics, ${ }^{12-14}$ the presence of secondary relaxations and "excess wings" and their connection to vitrification,,$^{3,15-19}$ and the dynamics of blends or block copolymers. ${ }^{20-22}$ The overriding goal of such studies is to relate chemical structure to the observed properties. The emergence of patterns of behavior enables correlations to be drawn, ${ }^{23-29}$ which in turn guide theoretical developments.

At a certain characteristic temperature, supercooled liquids and polymers exhibit various changes in their dynamics. ${ }^{30-32}$ These changes include a change in the shape of the relaxation function at a temperature, $T_{\mathrm{C}}$, a change in the temperature dependence of the relaxation times at $T_{\mathrm{B}},{ }^{33-36}$ and an extrapo-

* Address correspondence to this author.

$\dagger$ Silesian University.

$\doteqdot$ Naval Research Laboratory. lated merging at $T_{\beta}$ of the secondary relaxation with the primary $\alpha$-process. ${ }^{19,37,38}$ For a given glass former, the value of the $\alpha$-relaxation time at $T_{\mathrm{B}}$ has been shown to be a constant, independent of temperature and pressure. ${ }^{1,14,39}$

In this paper we describe dielectric measurements on two dibutyl phthalates, di- $n$-butyl phthalate (DBP) and diisobutyl phthalate (DiBP), whose molecular structures are illustrated in Figure 1. These are typical van der Waals liquids, and having substantial dipole moments, they can be easily probed with dielectric spectroscopy. Both liquids are slow to crystallize, and can be supercooled without the need for rapid quenching. A number of previous studies on DBT have been reported. The first measurements on DBT under elevated pressure were by Cook et al., ${ }^{40}$ who determined the viscosity at up to $3 \mathrm{GPa}$. At ambient pressure, Dufour et al. ${ }^{41}$ found no decoupling of the dielectric relaxation and the viscosity of DBP, while Menon and co-workers ${ }^{42}$ reported no decoupling of the viscosity and the mechanical shear modulus. This latter result was contradicted by further measurements by Behrens and co-workers. ${ }^{43} \mathrm{We}$ recently determined that the $\alpha$-relaxation and the ionic conductivity in DBP remain coupled at elevated pressures up to 1.6 $\mathrm{GPa} .{ }^{38}$ Measurements at lower pressure on DiBP suggested that the $\alpha$-relaxation times had roughly the same temperature dependence as determined by NMR and mechanical measurements. ${ }^{4,45}$ Herein, we describe results for the dielectric $\alpha$ - and $\beta$-relaxations, as well as the ionic conductivity, in both DBP and DiBP over a range of temperatures at pressures up to 1.8 GP. The isomers provide an interesting test of the effect of chemical structure on glass transition properties. We compare 
<smiles>CCCCOC(=O)c1ccccc1C(=O)OCCCC</smiles>

\section{di-n-butylphthalate (DBP)}<smiles>CC(C)COC(=O)c1ccccc1C(=O)OCC(C)C</smiles>

\section{di-isobutylphthalate (DiBP)}

Figure 1. Chemical structures of dibutyl phthalates.

the behavior of the two liquids, and in particular the variation with temperature and pressure.

\section{Experimental Section}

Di- $n$-butyl phthalate (99\%) and diisobutyl phthalate (99\%) were obtained from Aldrich Chemicals and used as received. The nominal glass transition temperatures, $T_{\mathrm{g}}$, are $179(\mathrm{DBP})^{42}$ and $188 \mathrm{~K}$ (DiBP). ${ }^{46}$ Measurements of the complex dielectric permittivity $\epsilon^{*}(\omega)=\epsilon^{\prime}(\omega)-i \epsilon^{\prime \prime}(\omega)$ were carried out with the Novo-Control GmbH Alpha dielectric spectrometer $\left(10^{-2}-10^{7}\right.$ $\mathrm{Hz}$ ), in combination with an Agilent 4291B impedance analyzer $\left(10^{6}-10^{9} \mathrm{~Hz}\right)$. For ambient pressure, the sample was placed in a parallel plate cell (diameter $20 \mathrm{~mm}$, gap $0.1 \mathrm{~mm}$ ), and the temperature controlled with a nitrogen-gas cryostat. Temperature stability was at least $0.1 \mathrm{~K}$.

For high-pressure measurements we used a pressure system constructed by Unipress (High Pressure Research, Poland), with the sample contained between two steel plates mounted in a Teflon bellows. Pressures up to $1.8 \mathrm{GPa}$ were exerted via a piston and hydraulic press. During measurements, the sample
TABLE 1: Relaxation Properties of Dibutyl Phthalates

\begin{tabular}{lccccc}
\hline & $T_{\mathrm{g}}{ }^{a}(\mathrm{~K})$ & $\beta_{\mathrm{K}}{ }^{b}$ & $m^{a}$ & $\begin{array}{c}\mathrm{d} T_{\mathrm{g}} / \mathrm{d} P^{a} \\
(\mathrm{~K} / \mathrm{MPa})\end{array}$ & $\begin{array}{c}\Delta V_{\alpha}{ }^{a} \\
(\mathrm{~mL} / \mathrm{mol})\end{array}$ \\
\hline DBP & $182.3 \pm 0.3$ & $0.65 \pm 0.1$ & $60 \pm 3$ & $0.11 \pm 0.01$ & 70 \\
DiBP & $196.8 \pm 1.1$ & $0.65 \pm 0.1$ & $56 \pm 3$ & $0.15 \pm 0.01$ & 103
\end{tabular}

${ }^{a}$ Ambient pressure and $\tau_{\alpha}=1 \mathrm{s.}{ }^{b} T>T_{\mathrm{B}}$.

was in contact only with the steel plates and Teflon insulation. The temperature was controlled within $0.1 \mathrm{~K}$ by means of liquid flow from a thermostatic bath.

\section{Results}

$\alpha$-Relaxation. In Figure 2 are shown representative dielectric loss curves for the $\alpha$-relaxation $\left(T>T_{\mathrm{g}}\right.$ ), and the $\beta$-process ( $T$ $<T_{\mathrm{g}}$ ) in DiBP at $T=295.7 \mathrm{~K}$ for various pressures from ambient up to $1.4 \mathrm{GPa}$. Toward the low-frequency side of the $\alpha$-peak is the contribution from ionic conductivity, $\sigma$. With increasing pressure, the $\alpha$-relaxation and $\sigma$ both shift to lower frequencies, while there is an almost negligible shift of the $\beta$-peak. The $\alpha$-dispersions measured for both materials were fit to the empirical Havriliak-Negami function ${ }^{47}$

$$
\epsilon^{\prime \prime}(v)=\operatorname{Im} \Delta \epsilon_{\mathrm{HN}} \frac{1}{\left(1+\left(i 2 \pi f \tau_{\mathrm{HN}}\right)^{\left.\alpha_{\mathrm{HN}}\right)^{\gamma_{\mathrm{HN}}}}\right.}
$$

where $f$ is the frequency in $\mathrm{Hz}, \Delta \epsilon_{\mathrm{HN}}$ is the relaxation strength, and $\alpha_{\mathrm{HN}}$ and $\gamma_{\mathrm{HN}}$ are shape parameters. The relaxation time in eq 1 is related to the $\tau_{\alpha}$ defined from the maximum in the dielectric loss $\left(\tau_{\alpha}=\left(2 \pi f_{\max }\right)^{-1}\right)$, according to ${ }^{34} \tau_{\mathrm{HN}}=\tau_{\alpha} \sin ^{1 / \alpha_{\mathrm{HN}-}}$ $\left(\alpha_{\mathrm{HN}} \pi / 2+2 \gamma_{\mathrm{HN}}\right) \sin ^{-1 / \alpha_{\mathrm{HN}}}\left(\alpha_{\mathrm{HN}} \gamma_{\mathrm{HN}} \pi / 2+2 \gamma_{\mathrm{HN}}\right)$. The best-fit values of the shape parameters are displayed in Figure 3. Approaching $T_{\mathrm{g}}$ from above, the $\alpha$-peak broadens until some characteristic value of the $\alpha$-relaxation time, $\tau_{\mathrm{c}}$, is attained. Beyond $\tau_{\mathrm{c}}$, the shape of the relaxation function becomes constant; that is, at lower temperatures/higher pressures, timetemperature-pressure superpositioning becomes valid. $T_{\mathrm{c}}$ is defined as the temperature at which $\tau_{\alpha}$ attains the value $\tau_{\mathrm{c}} . T_{\mathrm{c}}$ values for both isomers are collected in Table 2 For DiBP, $\tau_{\mathrm{c}}$ $\sim 1 \times 10^{-7} \mathrm{~s}$, while for DBP, the crossover to a temperaturedependent $\alpha$-function occurs at a somewhat larger value of $\tau_{\mathrm{c}}$ $\sim 3 \times 10^{-7} \mathrm{~s}$. For both glass formers, $\tau_{\mathrm{c}}$ appears to be invariant

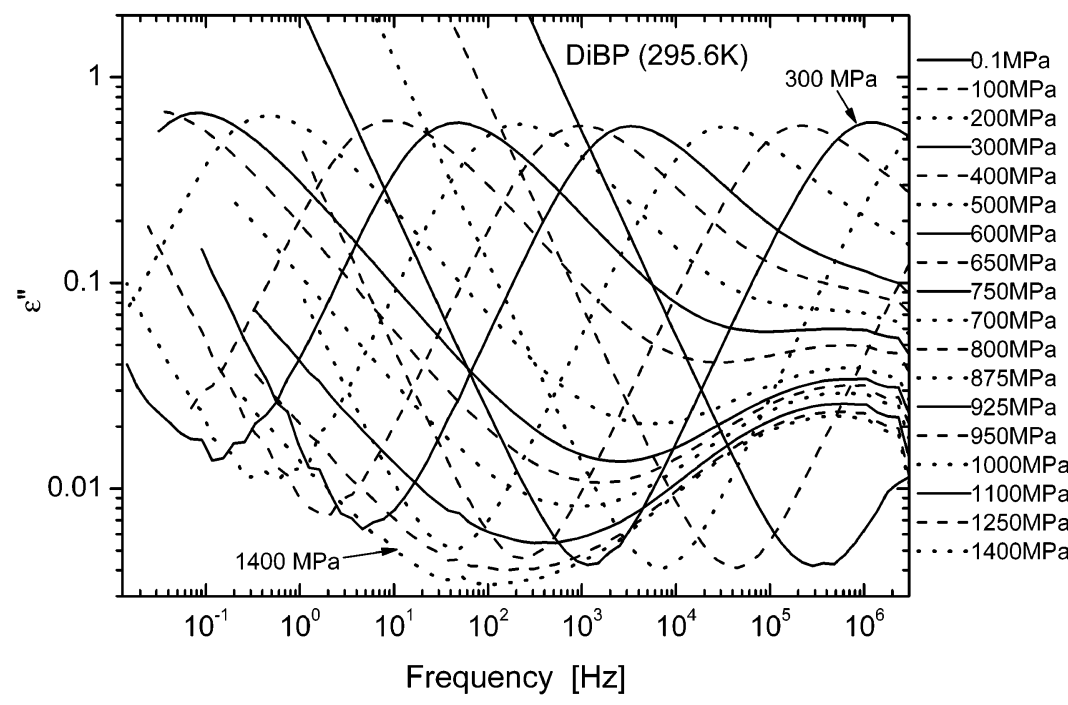

Figure 2. Representative dielectric loss curves for DiBP at $295.6 \mathrm{~K}$ and pressures from $0.1 \mathrm{MPa}$ to $1.4 \mathrm{GPa}$. The steep rise toward lower frequencies is due to ionic conductivity, while the secondary peak is evident at high frequencies in the spectra measured at higher pressures. 
TABLE 2: Characteristic Temperatures and Relaxation Times for Dibutyl Phthalates

\begin{tabular}{lllcccc}
\hline & $T_{\mathrm{C}}(\mathrm{K})$ & \multicolumn{1}{c}{$\log \tau_{\mathrm{c}}(\mathrm{s})$} & $T_{\mathrm{B}}{ }^{a}(\mathrm{~K})$ & $\log \tau_{\mathrm{B}}(\mathrm{s})$ & $T_{\beta}{ }^{a}(\mathrm{~K})$ & $\log \tau_{\beta}(\mathrm{s})$ \\
\hline DBP & $220 \pm 1$ & $(3 \pm 1) \times 10^{-7}$ & & & $229 \pm 2$ & $(7.8 \pm 0.6) \times 10^{-8}$ \\
DiBP & $243 \pm 0.5$ & $(1.2 \pm 0.6) \times 10^{-7}$ & $262.3 \pm 1.3$ & $7.7 \pm 1 \times 10^{-9}$ & $263.5 \pm 1.5$ & $(6.5 \pm 0.5) \times 10^{-9}$
\end{tabular}

${ }^{a}$ Ambient pressure.

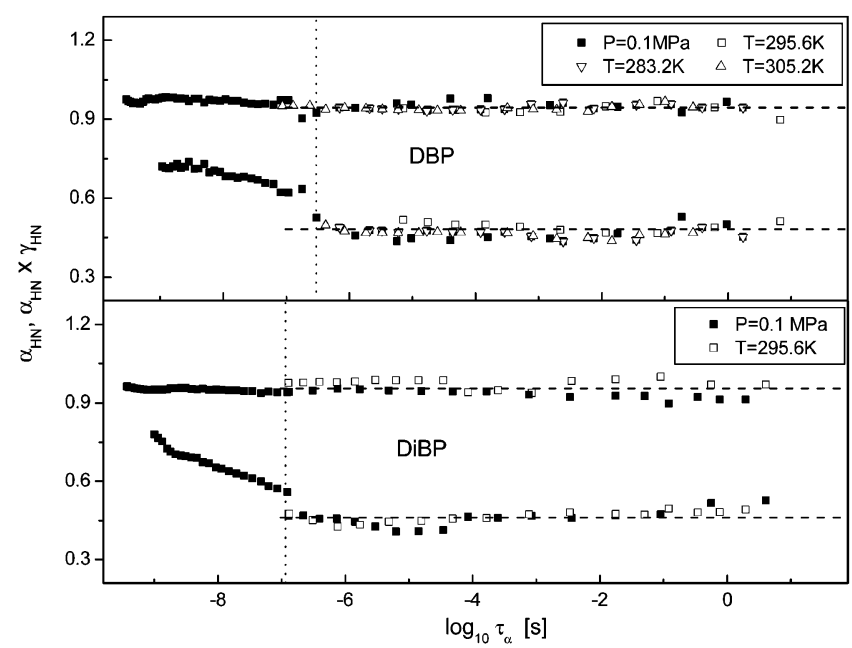

Figure 3. Fitting parameters for eq 1. Upper panel: DBP at various temperatures and ambient pressure (ם), and at various pressures and $T$ $=283.2(\nabla), 295.7(\square)$, and 305.2 $\mathrm{K}(\triangle)$. Lower panel: DiBP at various temperatures and ambient pressure (ם), and at various pressures and $T$ $=295.6 \mathrm{~K}(\square)$. The vertical dotted line denotes the value of $\tau_{\alpha}$ separating the two dynamical regimes. The horizontal dashed lines represent the mean values for lower temperatures and higher pressures: $\alpha_{\mathrm{HN}}=0.95 \pm 0.01, \alpha_{\mathrm{HN}} \times g_{\mathrm{HN}}=0.48 \pm 0.04$ for both liquids.

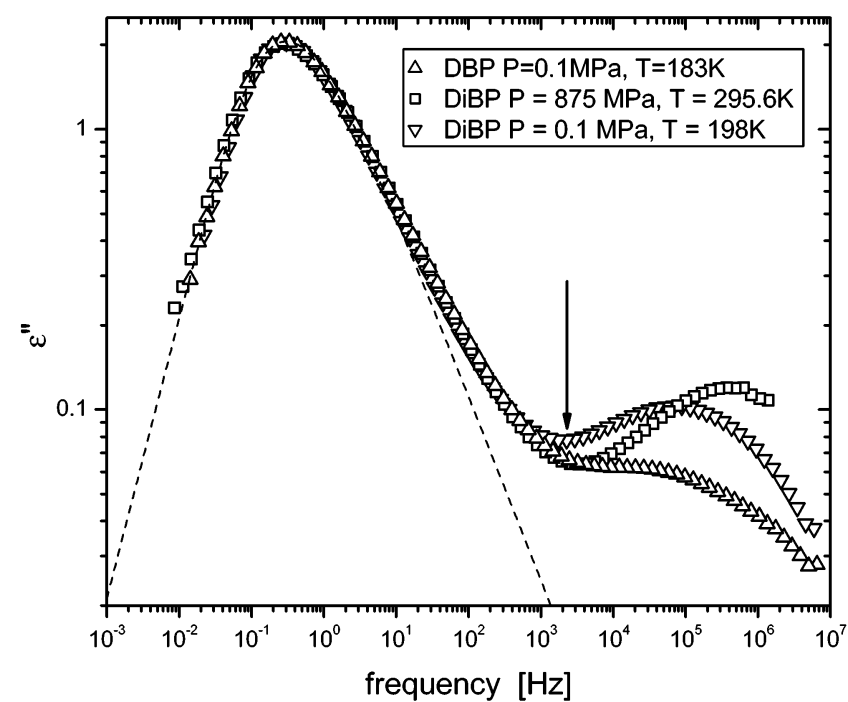

Figure 4. Dielectric loss of $\operatorname{DBP}(\triangle)$ and $\operatorname{DiBP}(\nabla, \square)$. Along with small vertical shifts, for the elevated pressure spectra, the frequencies were shifted by 1.1 for DBP and 0.6 for DiBP, to superpose the $\alpha$-dispersions. The dashed line is the Kohlrausch function with a stretch exponent equal to 0.66 . The secondary relaxation peaks at higher frequencies do not superpose. The arrow denotes the position of the Johari-Goldstein process predicted from eq 6.

to pressure. A similar result was reported previously for other molecular glass formers. ${ }^{1,14,39}$

The superpositioning of the $\alpha$-peaks for $\tau_{\alpha}>\tau_{\mathrm{c}}$ is illustrated in Figures 4 and 5, showing representative dielectric loss curves measured at various temperatures and pressures. In the supercooled regime, the two butyl phthalates also have the same shape parameters, $\alpha_{\mathrm{HN}}=0.95 \pm 0.01$ and $\gamma_{\mathrm{HN}}=0.50 \pm 0.04$. Fitting

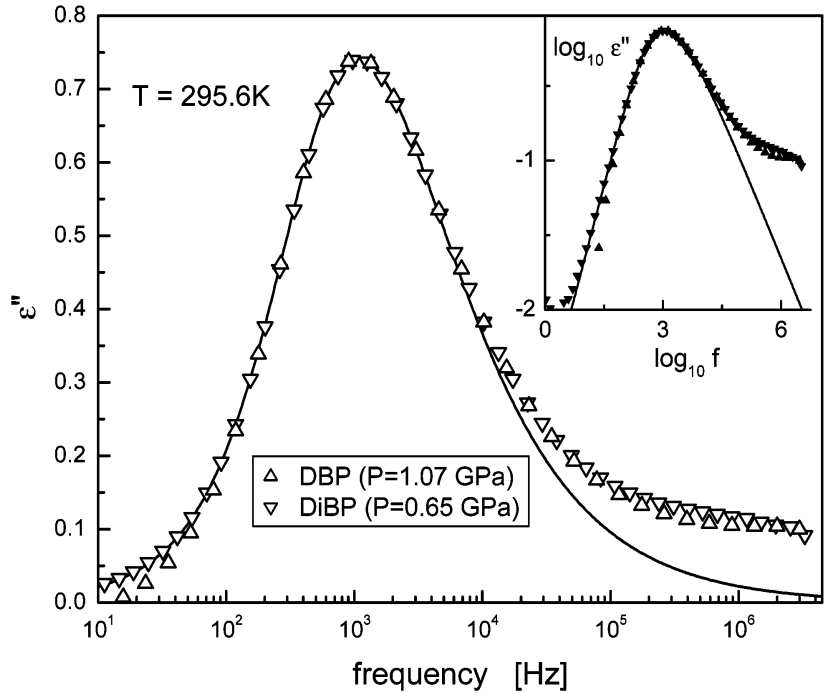

Figure 5. $\alpha$-Dispersion in the dielectric loss of DBP $(\triangle)$ and DiBP $(\nabla)$ at $295.6 \mathrm{~K}$ and $P=1.07$ and $0.65 \mathrm{GPa}$, respectively. The fitted curve is the Kohlrausch function with a stretch exponent equal to 0.65 . The contribution of the secondary relaxation is apparent at higher frequencies. The inset shows the same spectra plotted doublelogarithmically.

these peaks to the Kohlrausch-Williams-Watts function ${ }^{48}$

$$
\epsilon^{\prime \prime}(\omega)=\Delta \epsilon \int_{0}^{\infty} \mathrm{d} t\left[\frac{-\mathrm{d}}{\mathrm{d} t} \exp \left(-t / \tau_{\mathrm{K}}\right)^{\beta_{K}}\right] \sin (2 \pi f t)
$$

yields $\beta_{\mathrm{K}}=0.65 \pm 0.1$, as shown in Figures 4 and 5. (Note this is significantly larger than the value estimated from the relation of Alegria and Colmenero, $\left.{ }^{49} \beta_{\mathrm{K}}=\left(\alpha_{\mathrm{HN}} \gamma_{\mathrm{HN}}\right)^{0.813}\right)$. For this value of the stretch exponent, the Kohlrausch relaxation time in eq $2, \tau_{\mathrm{K}}$, is $20 \%$ smaller than $\tau_{\alpha}$ based on the peak frequency and $27 \%$ smaller than the average relaxation time.

The $\alpha$-relaxation times are displayed in Figure 6 for ambient pressure, along with fits to the Vogel-Fulcher (VF) equation ${ }^{50}$

$$
\tau_{\alpha}(T)=\tau_{0} \exp \left(\frac{B}{T-T_{0}}\right)
$$

We obtain $\tau_{0}=(3.4 \pm 0.2) \times 10^{-13} \mathrm{~s}, B=1016 \pm 5 \mathrm{~K}$, and $T_{0}=146.9 \pm 0.1 \mathrm{~K}$ for DBP and $\tau_{0}=(1.7 \pm 0.2) \times 10^{-14} \mathrm{~s}$, $B=1444 \pm 16 \mathrm{~K}$, and $T_{0}=151.3 \pm 0.4 \mathrm{~K}$ for DiBP. Using eq 3 to interpolate, we find the temperature at which $\tau_{\alpha}=1 \mathrm{~s}$, which we use herein as the glass transition temperature. The results are $T_{\mathrm{g}}=182.3 \pm 0.3$ and $196.8 \pm 1.1 \mathrm{~K}$ for $\mathrm{DBP}$ and DiBP, respectively. These are 3-9 deg higher than the literature values, ${ }^{42,46}$ since we are referencing to a small value of $\tau_{\alpha}$ to avoid any extrapolation of the measured data.

Since the shape of the $\alpha$-relaxation functions for DBP and DiBP is the same, from the correlation between $\beta_{\mathrm{K}}$ and the steepness index, $\left.m \equiv\left(\partial \log \tau_{\alpha}\left(T_{g}\right) / \partial\left(T_{g} / T\right)\right)_{p}\right|_{T=T_{g}},{ }^{51,52}$ established for a large number of low-molecular-weight glass-forming liquids, ${ }^{53,54}$ we expect $m$ to be the same for the two materials. In Figure 7, we replot the data of Figure 6 after normalizing the temperature by the respective $T_{\mathrm{g}}$ values. As can be seen, the fragilities for the $\alpha$-process are nearly equivalent, $m=60$ 


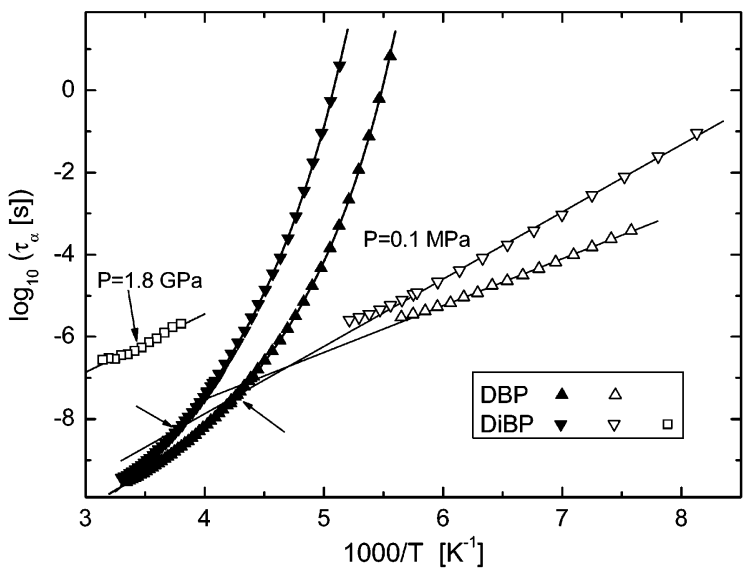

Figure 6. Temperature dependence of the $\alpha$-relaxation times of DBP $(\boldsymbol{\Delta})$ and $\operatorname{DiBP}(\boldsymbol{\nabla})$ and the corresponding secondary relaxation times (open symbols) at ambient pressure. The solid lines are the fits to eq 3 for $\tau_{\alpha}$ and the Arrhenius equation for $\tau_{\beta}$. Also shown for DiBP are the secondary relaxation times measured at $P=1.8 \mathrm{GPa}(\square)$. The arrows indicate the temperatures at which linear extrapolations of the respective ambient-pressure $\tau_{\beta}$ would intersect the $\tau_{\alpha}$ data, at $T_{\beta}=229$ and $263.5 \mathrm{~K}$, for DBP and DiBP, respectively.

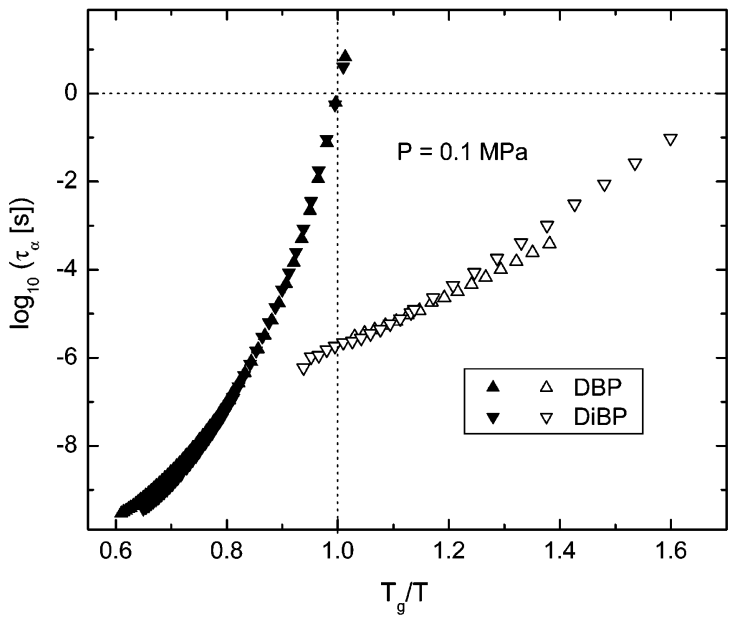

Figure 7. $T_{\mathrm{g}}$-normalized temperature dependence at ambient pressure of the $\alpha$-relaxation times of $\operatorname{DBP}(\boldsymbol{\Lambda})$ and $\operatorname{DiBP}(\boldsymbol{\nabla})$, along with the corresponding relaxation times of the secondary process (open symbols).

\pm 3 (DBP) and $56 \pm 3$ (DiBP). The former is consistent with the value of $m=69$ reported for DBP at lower temperature $\left(\tau_{\alpha}\left(T_{\mathrm{g}}\right)=100 \mathrm{~s}\right){ }^{42,53}$ These $m$ values for the two dibutyl phthalates yield equivalent activation energies at $T_{\mathrm{g}}, E_{\alpha}=$ $2.303 R T_{g} m=210 \pm 12 \mathrm{~kJ} / \mathrm{mol}$.

Representative results for $\tau_{\alpha}$ as a function of pressure are shown in Figure 8 at $T=295.6 \mathrm{~K}$. It can be seen that the sensitivity of the $\alpha$-process to pressure for DiBP is greater than that for DBP. This can be quantified in terms of the activation volume, defined as $\Delta V(T)=\left.2.303 R T\left(\partial \log \tau_{\alpha} / \partial P\right)\right|_{T}$, with the results shown in Figure 9. For $\tau_{\alpha}=1 \mathrm{~s}, \Delta V_{\alpha}$ equals $103 \mathrm{~mL} /$ mol for DiBP, versus $70 \mathrm{~mL} / \mathrm{mol}$ for DBP. In the inset, we show the activation volumes for various temperatures at $\tau_{\alpha}=$ $1 \mathrm{~s} . \Delta V_{\alpha}$ is larger for DiBP for all measured isotherms.

Increasing the pressure increases the glass transition temperature. In Figure 10, we plot the temperature at which $\tau_{\alpha}=1 \mathrm{~s}$ as a function of pressure. These data can be described by using the Avramov relation ${ }^{55,56}$

$$
T_{g}=a\left(1+\frac{b}{c} P\right)^{1 / b}
$$

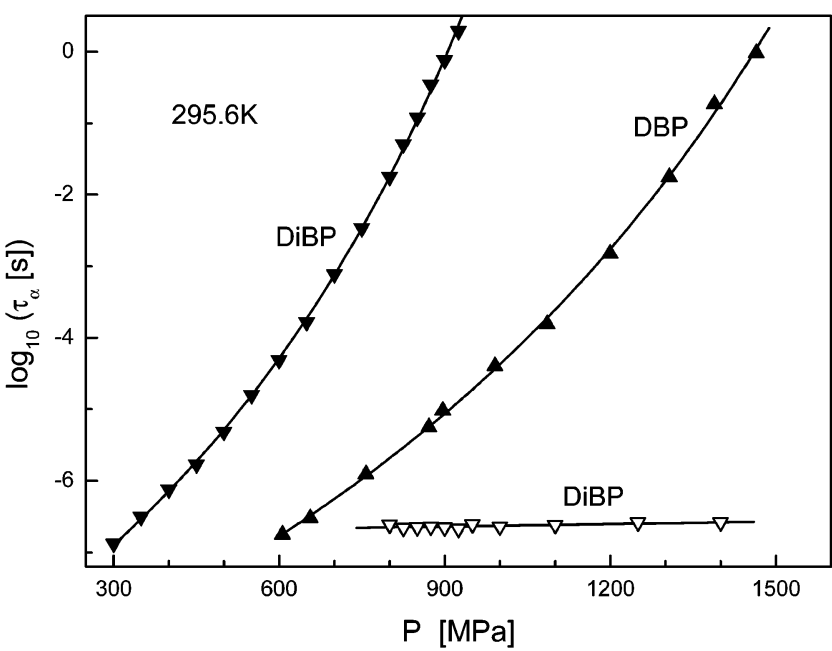

Figure 8. Pressure dependence of the $\alpha$-relaxation times of DBP $(\mathbf{\Delta})$ and $\operatorname{DiBP}(\boldsymbol{\nabla})$ at $295.6 \mathrm{~K}$ (solid lines are second-degree polynomial fits). Also shown are the secondary relaxation times for $\operatorname{DiBP}(\nabla)$ at the same temperature, with a linear fit corresponding to an activation volume of $0.7 \pm 0.2 \mathrm{~mL} / \mathrm{mol}$.

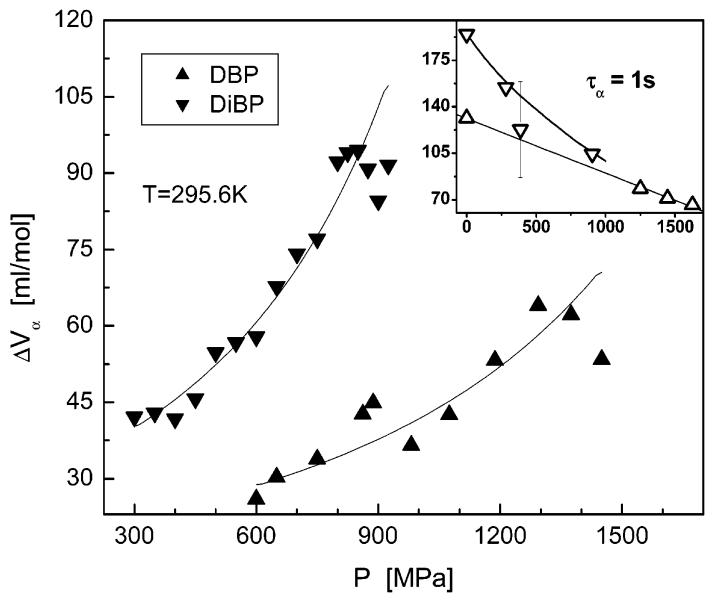

Figure 9. Activation volumes for the $\alpha$-relaxation of DBP $(\mathbf{\Lambda})$ and $\operatorname{DiBP}(\boldsymbol{\nabla})$ as a function of pressure at $295.6 \mathrm{~K}$. The inset shows activation volumes for $\operatorname{DBP}(\triangle)$ and $\operatorname{DiBP}(\nabla)$ versus pressure for a fixed value of the $\alpha$-relaxation time. The single datum with the large error bar required extrapolation to reach $\tau_{\alpha}=1 \mathrm{~s}$. For all other points, the error is smaller than the symbol size.

The results for DBP are $a=182.3 \pm 0.6 \mathrm{~K}, b=2.4 \pm 0.2$, and $c=1600 \pm 100 \mathrm{MPa}$, and for DiBP $a=197.5 \pm 2 \mathrm{~K}, b$ $=2.65 \pm 0.2$, and $c=1293 \pm 95 \mathrm{MPa}$. The pressure coefficient of the glass transition in the limit of zero pressure is $\mathrm{d} T_{\mathrm{g}} / \mathrm{d} P=$ $0.11 \pm 0.01$ and $=0.15 \pm 0.01 \mathrm{~K} / \mathrm{MPa}$ for DBP and DiBP, respectively. Again, $\mathrm{DiBP}$ is seen to be more sensitive to pressure.

The Avramov model underlying eq 4 embodies the assumption that the temperature and pressure dependences are factorable, whereby the fragility is independent of pressure. ${ }^{57} \mathrm{We}$ can make use of the pressure coefficients determined for $\tau_{\alpha}$ and $T_{\mathrm{g}}$ to calculate the variation of the fragility with pressure, using

$$
m=\frac{\Delta V_{\alpha}\left(T_{g}\right)}{2.303 R}\left(\frac{\mathrm{d} T_{g}}{\mathrm{~d} P}\right)^{-1}
$$

The results are displayed in Figure 11. The uncertainty is large, and thus we conclude only that there is no significant change in the temperature dependence of $\tau_{\alpha}$ with pressure. Similarly, from viscosities measured at elevated pressures, Cook et al. ${ }^{40}$ 


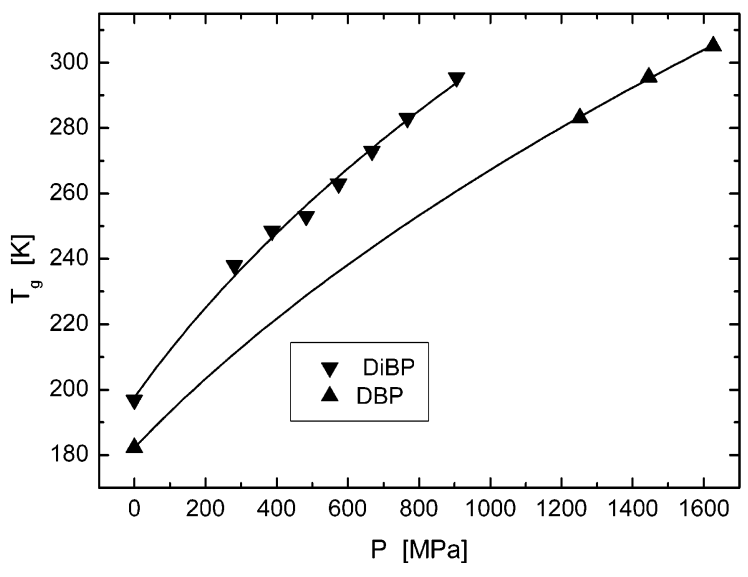

Figure 10. Pressure dependence of the glass transition temperature, $T_{\mathrm{g}}\left(\tau_{\alpha}=1 \mathrm{~s}\right)$ for $\operatorname{DBP}(\mathbf{\Delta})$ and $\operatorname{DiBP}(\boldsymbol{\nabla})$. The lines through the data are the best fits to the Avramov relation (eq 4).

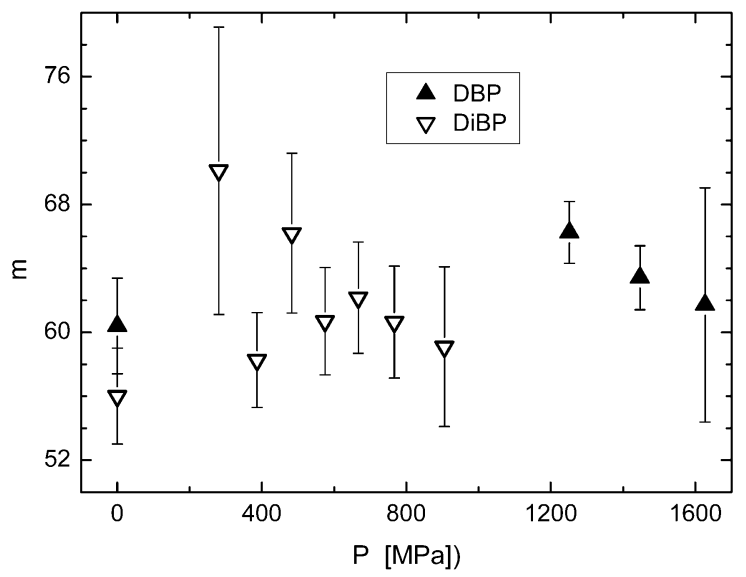

Figure 11. Pressure dependence of the fragility of the $\alpha$-relaxation for $\operatorname{DBP}(\mathbf{\Lambda})$ and $\operatorname{DiBP}(\boldsymbol{\nabla})$.

found that pressure caused no change in $m$ beyond the (rather large) experimental uncertainty. Similar results have been reported for other glass formers, ${ }^{58-61}$ although cases are known in which the $m$ decreases ${ }^{62,63}$ or increases ${ }^{11,18}$ with pressure.

Secondary Relaxation. Along with the ambient pressure $\tau_{\alpha}$ in Figure 6 are shown the corresponding relaxation times for the secondary relaxation, $\tau_{\beta}$, defined from the frequency of the secondary peak in the dielectric loss. Arrhenius behavior is observed, with respective activation energies, $E_{\beta}$, equal to 21.2 $\pm 0.7 \mathrm{~kJ} /(\mathrm{mol} \cdot \mathrm{deg})(\mathrm{DBP})$ and $30.2 \pm 0.8 \mathrm{~kJ} /(\mathrm{mol} \cdot \mathrm{deg})(\mathrm{DiBP})$ at $P=0.1 \mathrm{MPa}$. These are an order of magnitude smaller than $E_{\alpha}$ values at $T_{\mathrm{g}}$. Kudlik et al. ${ }^{15}$ have suggested a universal relationship, $E_{\beta}=24 R T_{\mathrm{g}}$. However, the dibutyl phthalates depart from the proposed proportionality, and even differ between each other; to wit, $E_{\mathrm{a}} /\left(R T_{\mathrm{g}}\right)=14.0 \pm 0.5(\mathrm{DBP})$ and $18.5 \pm 0.6$ (DiBP). However, as seen in Figure 7, the $T_{\mathrm{g}}$ normalization of the temperature variable does approximately superpose the ambient pressure $\tau_{\beta}$ from Figure 6 , although the $\beta$-relaxation times for DiBP have a steeper slope. Also included in Figure 6 are the $\tau_{\beta}$ measured for DiBP at $P=1.8 \mathrm{GPa}$. Over the narrow range of temperatures, the data are Arrhenius, with an activation energy equal to $28.9 \pm 1.8 \mathrm{~J} /(\mathrm{mol} \cdot \mathrm{deg})$. This is equal to $E_{\beta}$ for low pressure, within the uncertainty, and thus is also at odds with the proposed correlation ${ }^{15}$ between $E_{\beta}$ and $T_{\mathrm{g}}$, since $T_{\mathrm{g}}$ changes with pressure.

There is a suggestion in the $\tau_{\beta}$ data in Figure 6 of a deviation from the Arrhenius behavior on approaching $T_{\mathrm{g}}$. In this region, however, there is some overlapping of the $\alpha$ - and $\beta$-peaks, which makes the extraction of $\tau_{\beta}$ less certain. Nevertheless, it has been shown for at least a few cases that the Arrhenius temperature dependence of the $\tau_{\beta}$ changes for temperatures above $T_{\mathrm{g}}{ }^{19,64-68}$ Notwithstanding, it is common practice to extrapolate the lowtemperature secondary relaxation times to the temperature, $T_{\beta}$, at which $\tau_{\beta}=\tau_{\alpha}$. This hypothetical merging temperature is identified with the temperature at which a change in the dynamics is observed; that is, $T_{\beta} \sim T_{\mathrm{B}} \sim T_{\mathrm{C}} \cdot{ }^{30,32}$ From Figure $6, T_{\beta}=229 \mathrm{~K}$ for DBP and $263.5 \mathrm{~K}$ for DiBP.

At elevated pressure, obtaining $\tau_{\beta}$ requires deconvolution of the $\alpha$ - and $\beta$-processes. Although the error in $\tau_{\beta}$ is consequently large, it is nevertheless clear that the secondary relaxation times are much less sensitive to pressure than are the $\alpha$-relaxation times. In Figure 8, these $\tau_{\beta}$ are shown for DiBP, where it can be seen that over a pressure range for which $\tau_{\alpha}$ varies by 5 orders of magnitude, $\tau_{\beta}$ changes by only $20 \%$. This yields an estimate of the $\tau_{\beta}$ at ambient pressure equal to roughly $2 \times$ $10^{-7} \mathrm{~s}$. On the other hand, assuming that the temperature dependence of the secondary relaxation times at ambient pressure follows a single Arrhenius law, as depicted in Figure $6, \tau_{\beta}$ is predicted to be about two decades shorter than this. The implication is that the $\beta$-relaxation times in the liquid state may change more rapidly with pressure than in glass. Recent results have shown that the temperature dependences of $\tau_{\beta}$ above and below $T_{\mathrm{g}}$ are different. ${ }^{19,65-68}$ Even if the data follow a second Arrhenius form above $T_{\mathrm{g}}$, the activation energy will be larger for the liquid state.

In terms of the activation volume, $\Delta V_{\beta} \sim 0.7 \mathrm{~mL} / \mathrm{mol}$ for DiBP at $295.6 \mathrm{~K}$. For DBP, we estimate $\Delta V_{\beta}$ is about 10 -fold larger than that for DiBP. The weaker pressure sensitivity of $\tau_{\beta}$, in comparison to the $\alpha$-process, means that the timetemperature-pressure superpositioning of the dielectric spectra must break down. This is seen in Figure 4, in which shifting to superimpose the primary peak results in nonoverlapping of the higher frequency secondary peaks.

The almost negligible sensitivity to pressure of the secondary relaxation for either liquid raises the question of whether it is a Johari-Goldstein process, involving the entire molecule (rather than only intramolecular degrees of freedom). ${ }^{69}$ An assessment of this can be gleaned by comparing $\tau_{\beta}$ to the value predicted by the coupling model $^{70,71}$

$$
\tau_{\mathrm{JG}}=t_{\mathrm{c}}{ }^{1-\beta_{\mathrm{K}}} \tau_{\alpha}^{\beta_{\mathrm{K}}}
$$

in which $t_{\mathrm{c}}$ is a constant equal to $2 \times 10^{-12} \mathrm{~s}$. Using the fitted value of $\beta_{\mathrm{K}}=0.66$, we calculate $\tau_{\mathrm{JG}}=7 \times 10^{-12} \mathrm{~s}$, which is about a factor of 40 smaller than the $\tau_{\beta}$ value in Figure 4 . Thus, the inference drawn from the coupling model is that the secondary relaxation is not a Johari-Goldstein process.

Ionic Conductivity. Toward lower frequencies in the spectra of Figure 2, the contribution to the dielectric loss from ionic conductivity can be seen. Along with the slowing down of the $\alpha$-relaxation, lower temperature and increased pressure are associated with a decrease in the ionic conductivity. For many glass formers, translational motions are enhanced relative to orientations, at least below $T_{\mathrm{B}}$, so that $\tau_{\alpha}$ and $\sigma$ exhibit different $T$ and $P$ dependences. In Figure 12, $\sigma$ is plotted versus $\tau_{\alpha}$ for both materials at atmospheric pressure (the elevated pressure results had insufficient overlap of the two quantities). These data can be fitted to a power law ${ }^{72}$

$$
\sigma \tau^{s}=\text { constant }
$$

in which $s(\leq 1)$ reflects the degree of decoupling of $\tau_{\alpha}$ and $\sigma$. 


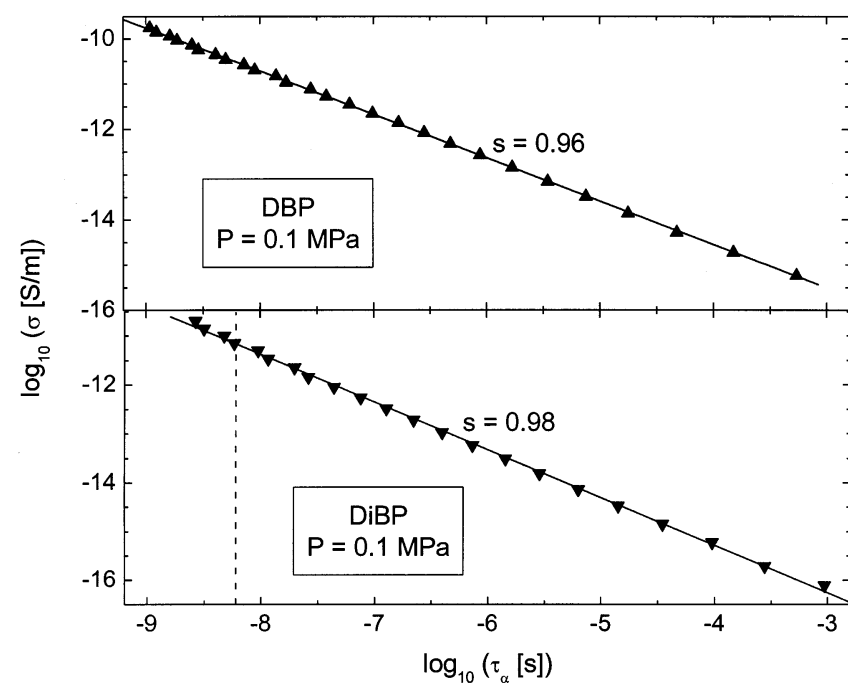

Figure 12. Ionic conductivity for $\operatorname{DBP}(\boldsymbol{\Lambda})$ and $\operatorname{DiBP}(\boldsymbol{\nabla})$ versus the $\alpha$-relaxation times measured at the same temperature and ambient pressure. The power law fits to the data yield the indicated values for the decoupling constant (eq 7). For DiBP $T_{\mathrm{B}}$ is indicated by the horizontal dashed line.

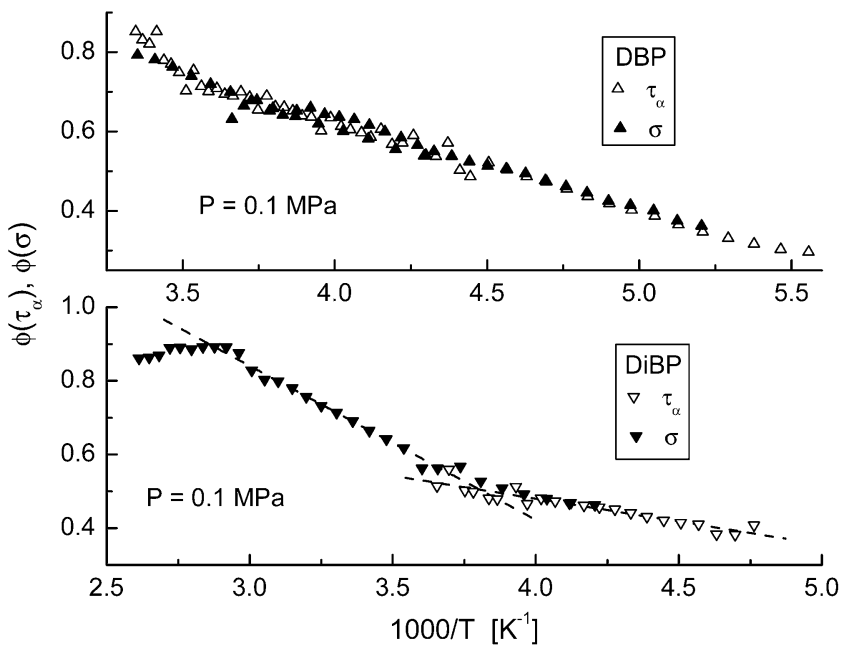

Figure 13. Derivative plots $\left(\phi \equiv \mathrm{d} \log x / T_{\mathrm{g}} \mathrm{d} T^{-1}\right)^{-1 / 2}$ of the $\alpha$-relaxation times (open symbols) and the ionic conductivity (solid symbols) for $\operatorname{DBP}(\mathbf{\Lambda}, \Delta)$ and $\operatorname{DiBP}(\boldsymbol{\nabla}, \nabla)$. The intersection of the dashed lines, which are linear fits to the two temperature regimes, yields $T_{\mathrm{B}}$. Note the approximate Arrhenius temperature dependence for DiBP for temperatures less than ca. $340 \mathrm{~K}$.

For both DBP and DiBP, the exponent is close to unity, indicating that $\tau_{\alpha}$ and $\sigma$ have essentially the same temperature dependence.

Since there is negligible decoupling of $\sigma$ and $\tau$, we can combine these data in a plot of $\phi \equiv\left(\operatorname{dlog} x / T_{\mathrm{g}} \mathrm{d} T^{-1}\right)^{-1 / 2}$, where $x$ is $\tau_{\alpha}$ or $\sigma$, versus inverse temperature. From such a derivative plot, shown in Figure 13 for both materials, the temperature, $T_{\mathrm{B}}$, associated with a change in temperature dependence, can be determined. ${ }^{34,35}$ However, for the DBP, any change in the slope of the derivative plot in Figure 13 is less than the scatter, precluding a determination of $T_{\mathrm{B}}$. For DiBP we obtain $T_{\mathrm{B}}=$ $262 \mathrm{~K}$. Note also that at the highest temperatures in Figure 13, the conductivity data for DiBP become horizontal, indicating that Arrhenius behavior is attained at ca. $343 \mathrm{~K}$.

\section{Conclusions}

Various phenomena involving the $\alpha$-relaxation, the $\beta$-relaxation, and the conduction of ionic impurities are observed herein for the two dibutyl phthalates. At ambient pressure, DBP and DiBP have very similar dynamics, beyond the $14.5 \mathrm{~K}$ difference in their $T_{\mathrm{g}}$ (Table 1). Both the shape of the $\alpha$-relaxation functions and the $T_{\mathrm{g}}$-normalized temperature dependences of $\tau_{\alpha}$ are essentially the same for the two isomers. A correlation of the breadth of the $\alpha$-peak and the fragility is expected. ${ }^{53,54} \mathrm{At}$ elevated pressure, differences between the dynamics of the two materials become apparent. The activation volume and pressure coefficient of $T_{\mathrm{g}}$ are significantly larger for the DiBP. These findings suggest the position of the pendant methyl group in DiBP enhances the steric hindrances to the motion, and consequently the dynamics of DiBP is more pressure sensitive. Such behavior, in which differences in the dynamics of structurally similar glass formers are manifested primarily at elevated pressures, has previously been observed for siloxane polymers. ${ }^{73}$

The activation energy for the secondary relaxation in DiBP is the same for $P=0.1 \mathrm{MPa}$ and $P=1.8 \mathrm{GPa}$. This invariance of $E_{\beta}$ to such a large increase in pressure reflects the local nature of the $\beta$-process. Moreover, the secondary relaxation in DiBP exhibits a very weak response to pressure $\left(\Delta V_{\beta}<1 \mathrm{~mL} / \mathrm{mol}\right)$. From this insensitivity, together with the magnitude of $\tau_{\beta}$ in comparison to the value calculated from eq 6 , it is tempting to suggest that the secondary peak evident in the dielectric spectrum is not a Johari-Goldstein process. Less information could be gleaned about the secondary process in DBP, other than its having a substantially larger activation volume than the $\Delta V_{\beta}$ for DiBP. For neither liquid does a proposed universal relationship ${ }^{15}$ between $E_{\beta}$ and $T_{\mathrm{g}}$ hold.

Decoupling of the $\alpha$-relaxation and conductivity commonly observed in supercooled liquids is ascribed to enhanced intermolecular cooperativity and the dynamic heterogeneity that it entails. Since the intermolecular cooperativity in these dibutyl phthalates is weak (large $\beta_{\mathrm{K}}$ ), ${ }^{74}$ it follows that the decoupling of their $\tau_{\alpha}$ and $\sigma$ should be weak. This is indeed the case, the temperature dependence of the two quantities remaining essentially equal at ambient pressure for all measured temperatures (Figure 12). This result is consistent with previous work showing that $\tau_{\alpha}$ and the viscosity of DBP conform to the Einstein-Stokes relation throughout the supercooled regime. ${ }^{38}$

The wide span of the data presented herein enables the changing dynamics exhibited during supercooling of the liquids to be followed. Thus, at the highest temperatures, $\tau_{\alpha}$ for DiBP exhibits an Arrhenius temperature dependence (as inferred from the conductivity data), with the molecules executing independent, thermally activated motion. From Figure 13, we estimate the characteristic temperature $T_{\mathrm{A}} \sim 343 \mathrm{~K}$, which is $\sim 1.7$ times $T_{\mathrm{g}}$, at which $\tau_{\alpha}<3 \times 10^{-11} \mathrm{~s}$. As temperature is lowered, or pressure increased, various changes in the dynamic properties become apparent. These changes include a broadening of the relaxation function between $T_{\mathrm{A}}$ and $T_{\mathrm{C}}$, a change in the temperature dependence of $\tau_{\alpha}$ at $T_{\mathrm{B}}$, and an apparent loss of the $\beta$-process as it merges with the primary $\alpha$-relaxation at $T_{\beta}$. Previous studies have suggested an approximate equivalence between these characteristic temperatures. As seen in Table 2, the values obtained herein for $T_{\mathrm{B}}$ and $T_{\beta}$ for DiBP roughly coincide. However, for both liquids, $T_{\mathrm{C}}<T_{\beta}$. Earlier studies ${ }^{1,14,39}$ had found that when pressure is varied, $T_{\mathrm{B}}$ changes in a manner such that the $\alpha$-relaxation time associated with the change in temperature dependence of $\tau_{\alpha}$ remains constant. The data herein cover an inadequate range to test this idea.

Acknowledgment. This work was supported by the Office of Naval Research and the Polish Committee for Scientific Research (Grant No. 2PO3B 033 23). 


\section{References and Notes}

(1) Casalini, R.; Paluch, M.; Roland, C. M. J. Chem. Phys. 2003, 118 , $5701-5703$

(2) Casalini, R.; Ngai, K. L.; Roland, C. M. Phys. Rev. B 2003, 68 , 014201 .

(3) Roland, C. M.; Casalini, R.; Paluch, M. Chem. Phys. Lett. 2003, $367,259-264$.

(4) Cailliaux, A.; Alba-Simionesco, C.; Frick, B.; Willner, L.; Goncharenko, I. Phys. Rev. E 2003, 67, 010802.

(5) Patkowski, A.; Lopes, M. M.; Fischer, E. W. J. Chem. Phys. 2003, $119,1579-1585$.

(6) Kamath, S.; Colby, R. H.; Kumar, S. K.; Baschnagel, J. J. Chem. Phys. 2002, 116, 865-868.

(7) Williams, G. In Dielectric Spectroscopy of Polymeric Materials; Runt, J. P., Fitzgerald, J. J., Eds.; American Chemical Society: Washington, DC, 1997.

(8) Paluch, M.; Roland, C. M.; Casalini, R.; Meier, G.; Patkowski, A. J. Chem. Phys. 2003, 118, 4578-4582.

(9) Paluch, M.; Casalini, R.; Roland, C. M. Phys. Rev. B 2002, 66 , 092202.

(10) Roland, C. M.; Casalini, R. Macromolecules 2003, 36, 1361-1367.

(11) Roland, C. M.; Casalini, R.; Santangelo, P.; Sekula, M.; Ziolo, J.; Paluch, M. Macromolecules 2003, 36, 4954-4959.

(12) Corezzi, S.; Fioretto, D.; Rolla, P. Nature 2002, 420, 653-656.

(13) Richert, R.; Angell, C. A. J. Chem. Phys. 1998, 108, 9016-9026.

(14) Casalini, R.; Paluch, M.; Fontanella, J. J.; Roland, C. M. J. Chem. Phys. 2002, 117, 4901-4906.

(15) Kudlik, A.; Tschirwitz, C.; Blochowicz, T.; Benkhof, S.; Rossler, E. J. Non-Cryst. Solids 1998, 235, 406-411.

(16) Hensel-Bielowka, S.; Paluch, M.; Ziolo, J.; Roland, C. M. J. Phys. Chem. B 2002, 106, 12459-12463.

(17) Casalini, R.; Roland, C. M. Phys. Rev. Lett. 2003, 91, 015702.

(18) Paluch, M.; Casalini, R.; Hensel-Bielowka, S.; Roland, C. M. J. Chem. Phys. 2002, 116, 9839-9844.

(19) Paluch, M.; Roland, C. M.; Pawlus, S.; Ziolo, J.; Ngai, K. L. Phys. Rev. Lett. 2003, 91, 115701.

(20) Floudas, G.; Fytas, G.; Reisinger, T.; Wegner, G. J. Chem. Phys. 1999, 111, 9129-9132.

(21) Alegria, A.; Gomez, D.; Colmenero, J. Macromolecules 2002, 35, 2030-2035.

(22) Zhang, S. H.; Casalini, R.; Runt, J.; Roland, C. M. Macromolecules 2003, 36, 9917-9923.

(23) Roland, C. M.; Nagi, K. L. Macromolecules 2000, 33, 3184-3184.

(24) Santangelo, P. G.; Ngai, K. L.; Roland, C. M. Macromolecules 1996, 29, 3651-3653.

(25) Roland, C. M. Macromolecules 1992, 25, 7031-7036.

(26) Ngai, K. L.; Roland, C. M. J. Polym. Sci., Part B: Polym. Phys. 1997, 35, 2503-2510.

(27) Matsuoka, S.; Quan, X. J. Non-Cryst. Solids 1991, 131, 293-301.

(28) Matsuoka, S.; Quan, X. Macromolecules 1991, 24, 2770-2779.

(29) Chao, Y. L.; Aklonis, J. J. J. Appl. Phys. 1984, 56, 2383-2385.

(30) Gotze, W.; Sjogren, L. Rep. Prog. Phys. 1992, 55, 241-376.

(31) Ngai, K. L.; Leon, C. J. Non-Cryst. Solids 2003, 315, 124-133.

(32) Ngai, K. L.; Roland, C. M. Polymer 2002, 43, 567-573.

(33) Ngai, K. L.; Magill, J. H.; Plazek, D. J. J. Chem. Phys. 2000, 112, 1887-1892.

(34) Stickel, F.; Fischer, E. W.; Richert, R. J. Chem. Phys. 1996, 104, 2043-2055.

(35) Stickel, F.; Fischer, E. W.; Richert, R. J. Chem. Phys. 1995, 102, 6251-6257.

(36) Leon, C.; Ngai, K. L. J. Phys. Chem. B 1999, 103, 4045-4051.

(37) Rault, J. J. Non-Cryst. Solids 2000, 271, 177-217.

(38) Paluch, M.; Sekula, M.; Pawlus, S.; Rzoska, S. J.; Ziolo, J.; Roland, C. M. Phys. Rev. Lett. 2003, 90, 175702 .
(39) Casalini, R.; Paluch, M.; Roland, C. M. J. Phys. Condens. Matter 2003, 15, S859-S867.

(40) Cook, R. L.; King, H. E.; Herbst, C. A.; Herschbach, D. R. J. Chem. Phys. 1994, 100, 5178-5189.

(41) Dufour, J.; Jorat, L.; Bondeau, A.; Siblini, A.; Noyel, G. J. Mol. Liq. 1994, 62, 75 .

(42) Menon, N.; Nagel, S. R.; Venerus, D. C. Phys. Review Lett. 1994, 73, 963-966.

(43) Behrens, C. F.; Christiansen, T. G.; Christensen, T.; Dyre, J. C.; Olsen, N. B. Phys. Rev. Lett. 1996, 76, 1553-1553.

(44) Suchanski, W.; Jurga, S.; Pakula, T.; Paluch, M.; Ziolo, J. J. Phys. Condens. Matter 2000, 12, 9551-9562.

(45) Paluch, M.; Ziolo, J.; Rzoska, S. J.; Habdas, P. Phys. Rev. E 1996, $54,4008-4010$

(46) Carpenter, M. R.; Davies, D. B.; Matheson, A. J. J. Chem. Phys. 1967, 46, 2451. 89 .

(47) Havriliak, S.; Negami, S. J. Polym. Sci. Polym. Symp. 1966, 14,

(48) Williams, G.; Watts, D. C. Trans. Faraday Soc. 1970, 66, 80.

(49) Alvarez, F.; Alegria, A.; Colmenero, J. Phys. Rev. B 1991, 44, $7306-7312$

(50) Ferry, J. D. Viscoelastic Properties of Polymers; Wiley: New York, 1980.

(51) Oldekop, V. W. Glastech. Ber. 1957, 30, 8-14.

(52) Angell, C. A. J. Non-Cryst. Solids 1991, 131, 13-31.

(53) Bohmer, R.; Ngai, K. L.; Angell, C. A.; Plazek, D. J. J. Chem. Phys. 1993, 99, 4201-4209.

(54) Plazek, D. J.; Ngai, K. L. Macromolecules 1991, 24, 1222-1224.

(55) Andersson, S. P.; Andersson, O. Macromolecules 1998, 31, 29993006.

(56) Paluch, M.; Roland, C. M. J. Non-Cryst. Solids 2003, 316, 413417.

(57) Avramov, I. J. Non-Cryst. Solids 2000, 262, 258-263.

(58) Paluch, M.; Roland, C. M.; Gapinski, J.; Patkowski, A. J. Chem. Phys. 2003, 118, 3177-3186.

(59) Paluch, M.; Roland, C. M.; Pawlus, S. J. Chem. Phys. 2002, 116, 10932-10937.

(60) Casalini, R.; Paluch, M.; Roland, C. M. J. Phys. Chem. A 2003, 107, 2369-2373.

(61) Paluch, M.; Roland, C. M.; Best, A. J. Chem. Phys. 2002, 117, $1188-1193$

(62) Roland, C. M.; Paluch, M.; Rzoska, S. J. J. Chem. Phys. 2003 , $119,12439-12441$

(63) Huang, D. H.; Colucci, D. M.; McKenna, G. B. J. Chem. Phys. 2002, 117, 7390-7390

(64) Olsen, N. B. J. Non-Cryst. Solids 1998, 235, 399-405.

(65) Olsen, N. B.; Christensen, T.; Dyre, J. C. Phys. Rev. E 2000, 62, $4435-4438$

(66) Wagner, H.; Richert, R. J. Phys. Chem. B 1999, 103, 4071-4077.

(67) Nozaki, R.; Zenitani, H.; Minoguchi, A.; Kitai, K. J. Non-Cryst. Solids 2002, 307, 349-355.

(68) Fujima, T.; Frusawa, H.; Ito, K. Phys. Rev. E 2002, 66, 031503. 6812

(69) Johari, G. P.; Pathmanathan, K. J. Chem. Phys. 1986, 85, 6811-

(70) Leon, C.; Ngai, K. L.; Roland, C. M. J. Chem. Phys. 1999, 110, $11585-11591$.

(71) Ngai, K. L. J. Chem. Phys. 1998, 109, 6982-6994.

(72) Corezzi, S.; Campani, E.; Rolla, P. A.; Capaccioli, S.; Fioretto, D. J. Chem. Phys. 1999, 111, 9343-9351.

(73) Paluch, M.; Pawlus, S.; Roland, C. M. Macromolecules 2002, 35, $7338-7342$

(74) Ngai, K. L.; Roland, C. M. Macromolecules 1993, 26, 6824-6830. 